\title{
MHC class II genotype and the control of viremia in HIV-1-infected individuals on highly active antiretroviral therapy
}

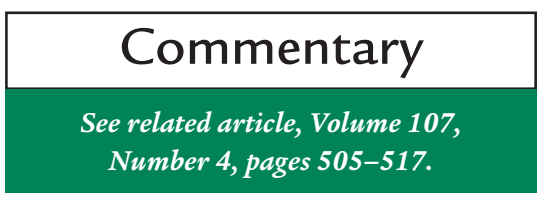

Joel N. Blankson and Robert F. Siliciano

Johns Hopkins University School of Medicine, Department of Medicine, Baltimore, Maryland, USA

Address correspondence to: Robert F. Siliciano, Johns Hopkins University School of Medicine, Department of Medicine, 1049 Ross Building, 720 Rutland, Baltimore, Maryland 21205, USA. Phone: (410) 955-2958; Fax: (410) 955-0964; E-mail: rsilicia@welch.jhu.edu.

HIV-1 infection sets up a complex dynamic equilibrium between viral replication and the HIV-1-specific immune response. In the absence of antiretroviral therapy, the high rate of viral replication, the associated depletion of $\mathrm{CD}^{+}{ }^{+} \mathrm{T}$ cells, and the high mutation rate of the virus combine to eventually overwhelm the immune system. In certain cases, however, it appears that the immune system has the upper hand, and viral replication occurs at only very low levels. Two groups of individuals fall into this category: some long-term nonprogressors (LTNPs) who have either very low or undetectable levels of plasma virus (1-3), and patients who are treated with highly active antiretroviral therapy (HAART) shortly after seroconversion who subsequently discontinue therapy $(4,5)$.

Much recent effort has been directed toward studying the HIV-specific immune response of these subjects in the hope of finding clues that will be helpful in designing vaccines. Viral antigen-specific $\mathrm{CD}^{+}{ }^{+}$and $\mathrm{CD}^{+} \mathrm{T}$ cells play an important role in HIV-specific immunity. The receptors on these cells recognize processed viral peptides that are presented by $\mathrm{MHC}$ molecules on the surfaces of antigen presenting cells. While considerable progress has been made regarding the mechanism by which responding $\mathrm{T}$ cells control viral replication, less is known about the role of the MHC genotype in patients who spontaneously control viremia.

\section{Class I MHC polymorphisms and disease progression}

MHC molecules are extremely polymorphic proteins that determine which processed peptides are presented to $T$ cells. Recent studies have shown a link between MHC expression and the rate of progression of HIV-1 infection. HLA $B * 57$ is one of three MHC class I alleles found to be independently associated

with slow progression of HIV-1 disease in a study of two large cohorts (6). Another recent study confirmed the high frequency of HLA B*57, and particularly the HLA B*5701 allele, in a subset of LTNPs with undetectable viral loads (3). Eleven out of 13 such individuals were HLA $B * 5701^{+}$whereas only 19 of 200 patients with progressive disease expressed this allele.

This association with class I molecules suggested that $\mathrm{CD}^{+}$cytotoxic $\mathrm{T}$ lymphocytes (CTLs) might play a role in slowing disease progression. Work by Goulder and colleagues demonstrated that the HIV-1-specific CTL response in these LTNPs was largely limited to HLA B*57-restricted viral epitopes (7). Migueles and colleagues confirmed this finding (3). Interestingly, these authors also showed that, whereas HLA B*5701+ individuals with progressive disease had as many HIV1-specific $\mathrm{CD}^{+} \mathrm{T}$ cells as did LTNPs, CTL responses in progressors were

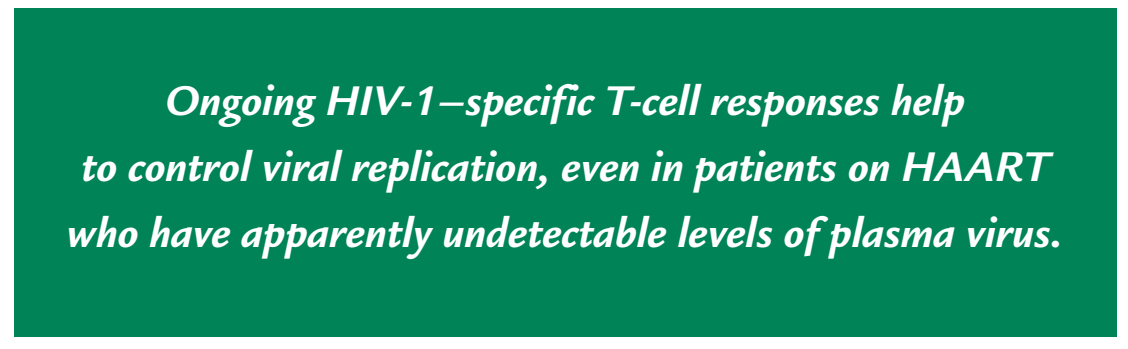

often mediated by other class I MHC molecules; in LTNP subjects, these responses were more often associated with HLA B*5701-restricted epitopes (3). This suggests that the ability to focus the CTL response to HLA B*5701-associated epitopes promotes spontaneous control of viremia, perhaps because of differences in antigen processing and/or the T-cell receptor repertoire in LTNPs.
The apparent advantage of a narrowly restricted T-cell response is counterintuitive, especially in light of recent data from Carrington et al. (8) showing a correlation between heterozygosity at the three class I loci and delayed onset of AIDS following seroconversion. Multiple MHC alleles, which would increase the diversity of the peptides that were presented to $T$ cells and result in a broad immune response, thus appear to be beneficial. In contrast, the work of Migueles and colleagues (3) suggests that a preventative vaccine consisting of peptides containing the identified haplotype-restricted epitopes would theoretically be superior to one containing conventional whole protein antigens in HLA B* $57^{+}$individuals.

\section{T-helper responses and MHC class II polymorphisms}

HIV-1-specific proliferative CD4 responses, which are largely absent in patients with chronic progressive dis-

ease, are seen in LTNPs $(1,2)$, suggesting that MHC class II molecules play at least an indirect role in the immune system's ability to control viremia. However, until recently, this role remained largely undefined. Chen and colleagues looked at the effect of HLA alleles on the rate of progression of disease in children with vertically transmitted HIV-1 infection. The HLA DR13 allele was present in 16 of 36 
children with slow progression but absent in all 14 children with rapid progression (9). In addition, in a recent study of three adult cohorts, the HLA DRB1*1300-DQB1*0603 haplotype was the only class II genotype associated with slow progression (10).

In this issue of the JCI, Malhotra and colleagues help clarify the role of this allele in HIV-1-specific immunity (11). The authors followed a cohort of 21 acute seroconverters who were treated with HAART shortly after presentation. Such early treatment has been shown to preserve HIV-1-specific CD4 proliferative responses. Interestingly, all seven patients with the HLA DRB1*13-DQB1*06 haplotype maintained suppression of viremia to below the limit of detection of current ultrasensitive assays (50 copies of HIV-1 $\mathrm{RNA} / \mathrm{ml}$ ) on HAART over an 18month period, compared with only 3 of 14 patients lacking this haplotype. As in prior studies $(2,5,12)$, all 21 patients were shown to have specific CD4 proliferative responses to the HIV-1 gag protein $\mathrm{p} 24$, but the responses were significantly stronger in the seven HLA DRB1*13-DQB $1 * 06+$ individuals. The results strongly suggest that the immune system continues to play a role in containing viremia in patients on HAART. This is a novel and surprising finding, as prior studies had shown a decline in the activity of HIV-1-specific $\mathrm{CD}^{+} \mathrm{T}$ cells (13-15) and had suggested a decline in the frequency of gag-specific $\mathrm{CD}^{+} \mathrm{T}$ cells in chronically infected patients on HAART (16). The data suggest that boosting the immune response through therapeutic vaccination may help maintain the efficacy of HAART.

\section{Identifying the relevant class II-restricted epitopes}

Having thus demonstrated the influence of the DRB1*13-DQB1*06 haplotype on therapeutic outcome, Malhotra and colleagues (11) went on to map two DRB1*13-restricted epitopes, using p24-specific T-cell clones. The peptide gag 26 (amino acids 251-270), which contained two distinct epitopes, was found to bind with reasonably high affinity to 10 of 12 DRB1 molecules tested. The best binding, however, was to DRB1*13. Another epitope was found in the peptide gag 30 (amino acids 291-310), which is located in a very highly conserved region of the protein. ELISPOT analysis demonstrated that more than $50 \%$ of cells from $\mathrm{p} 24$-specific CD4 lines, generated from four DRB ${ }^{*} 13^{+}$acute seroconverters, responded to one or both of these peptides, suggesting that they contain immunodominant epitopes.

Finally, the authors showed that this haplotype was present in 9 of 18 LTNPs and that LTNPs with this haplotype have significantly stronger $\mathrm{p} 24$-specific $\mathrm{CD}^{+}$proliferative responses than those lacking it (11). The authors suggest that strong immune responses to the immunodominant DRB1*13-restricted epitopes found in the $\mathrm{p} 24$ protein lead to control of viremia. The high affinity of gag 26 for HLA DRB1*13 results in better antigen presentation and hence a better immune response in patients expressing this allele. Therapeutic vaccination with peptide analogs of gag 26 , engineered to bind with higher affinity to other HLA DR molecules, might therefore improve the immune response and block disease progression in individuals expressing other haplotypes.

It should, however, be noted that the current study examined only p24 responses. LTNPs have been shown to have proliferative responses to other HIV-1 antigens including $\mathrm{p} 17, \mathrm{gp} 120$, and gp $66(1,17)$. It is possible that HLA DRB1*13-restricted immunodominant epitopes present in these other molecules are equally as important in containing viremia as the two found in the p24 protein. If so, then vaccination with multiple peptides and/or peptide analogs may be needed. The epitopes found in $\mathrm{p} 24$ contained what appears to be an HLA DRB1*13-specific binding motif, with either tyrosine or isoleucine at position 1 and either arginine or glycine at position 4.

\section{Prospects for fine-tuning HAART}

The study of Malhotra et al. (11) has important clinical implications and should also lead to a better understanding of HIV-1 pathogenesis. In addition to the vaccination strategies outlined above, the data may be useful in stratifying patients who are to be treated with HAART. Since patients lacking the HLA DRB1*13 allele appear to be less likely to maintain complete viral suppression on a standard regimen, it may be beneficial to treat such patients with more potent cocktails of antiretrovirals. Given the toxicity of these drugs, such a strategy may be helpful in optimizing the risk-to-benefit ratio in individual patients.

Peptides containing immunodominant epitopes can be used to construct tetramers with HLA DRB1*13 molecules. These reagents could then be used as probes to monitor $\mathrm{p} 24$-specific $\mathrm{CD}^{+}$ $\mathrm{T}$ cells in patients with this haplotype. Physical deletion of these cells can be distinguished from functional anergy in chronically infected patients with progressive disease. In addition, the emergence of viral escape mutants has been demonstrated in infected patients who had previously controlled viremia with strong CTL responses (18-20). It would thus be instructive to look for mutations in the p24 immunodominant epitopes in HLA DRB $1 * 13^{+}$LTNPs who eventually develop progressive disease. Most importantly, the exciting idea that ongoing HIV-1-specific T-cell responses help to control viral replication, even in patients on HAART who have apparently undetectable levels of plasma virus, needs further study to determine whether immunologic interventions could prolong responses to this therapy.

\section{Acknowledgments}

We thank Stuart Ray (Johns Hopkins University School of Medicine) for helpful discussions regarding epitope motifs.

1. Schwartz, D., et al. 1994. Absence of recoverable infectious virus and unique immune responses in an asymptomatic $\mathrm{HIV}^{+}$long-survivor. AIDS Res. Hum. Retroviruses. 10:1703-1711.

2. Rosenberg, E.S., et al. 1997. Vigorous HIV-1-specific $\mathrm{CD}^{+}{ }^{+} \mathrm{T}$ cell responses associated with control of viremia. Science. 278:1447-1450.

3. Migueles, S.A., et al. 2000 . HLA B*5701 is highly associated with restriction of virus replication in a subgroup of HIV-infected long term nonprogressors. Proc. Natl. Acad. Sci. USA. 97:2709-2714.

4. Lisziewicz, J., et al. 1999. Control of HIV despite the discontinuation of antiretroviral therapy. $N$. Engl. J. Med. 340:1683-1684.

5. Rosenberg, E.S., et al. 2000. Immune control of HIV-1 after early treatment of acute infection. Nature. 407:523-526.

6. Kaslow, R.A., et al. 1996. Influence of combinations of human major histocompatibility complex genes on the course of HIV-1 infection. Nat. Med. 2:405-411.

7. Goulder, P.J., et al. 1996. Novel, cross-restricted, conserved, and immunodominant cytotoxic T lymphocyte epitopes in slow progressors in HIV type 1 infection. AIDS Res. Hum. Retroviruses. 12:1691-1698.

8. Carrington, M., et al. 1999. HLA and HIV-1: heterozygote advantage and $\mathrm{B} * 35-\mathrm{CW}^{*} 04$ disadvantage. Science. 283:1748-1752.

9. Chen, Y., et al. 1997. Influence of HLA alleles on the rate of progression of vertically transmitted HIV infection in children: association of several HLA-DR13 alleles with long-term survivorship and the potential association of HLA-A*2301 with rapid progression to AIDS. Long-Term Survivor Study. Hum. Immunol. 55:154-162.

10. Keet, I.P., et al. 1999. Consistent associations of 
HLA class I and II and transporter gene products with progression of human immunodeficiency virus type 1 infection in homosexual men. J. Infect. Dis. 180:299-309.

11. Malhotra, U., et al. 2001. Role for HLA class II molecules in HIV-1 suppression and cellular immunity following antiretroviral treatment. J. Clin. Invest. 107:505-517.

12. Malhotra, U., et al. 2000. Effect of combination antiretroviral therapy on T-cell immunity in acute human immunodeficiency virus type 1 infection. J. Infect. Dis. 181:121-131.

13. Ogg, G.S., et al. 1998. Quantitation of HIV-1-specific cytotoxic $\mathrm{T}$ lymphocytes and plasma load of viral RNA. Science. 279:2103-2106.
14. Ogg, G.S., et al. 1999. Decay kinetics of human immunodeficiency virus-specific effector cytotoxic $\mathrm{T}$ lymphocytes after combination antiretroviral therapy. J. Virol. 73:797-800.

15. Kalams, S.A., et al. 1999. Levels of human immunodeficiency virus type 1 -specific cytotoxic T-lymphocyte effector and memory responses decline after suppression of viremia with highly active antiretroviral therapy. J. Virol. 73:6721-6728.

16. Pitcher, C.J., et al. 1999. HIV-1-specific CD4 $4^{+}$ cells are detectable in most individuals with active HIV-1 infection, but decline with prolonged viral suppression. Nat. Med. 5:518-525.

17. Blankson, J.N., Gallant, J.E., and Siliciano, R. 2001. Proliferative responses to HIV-1 antigens in
HIV-1 infected patients with immune reconstitution. J. Infect. Dis. In press.

18. Borrow, P., et al. 1997. Antiviral pressure exerted by HIV-1-specific cytotoxic T lymphocytes (CTLs) during primary infection demonstrated by rapid selection of CTL escape virus. Nat. Med. 3:205-211.

19. Goulder, P.J., et al. 1997. Late escape from an immunodominant cytotoxic T-lymphocyte response associated with progression to AIDS. Nat. Med. 3:212-217.

20. Price, D.A., et al. 1997. Positive selection of HIV1 cytotoxic T lymphocyte escape variants during primary infection. Proc. Natl. Acad. Sci. USA. 94:1890-1895. 